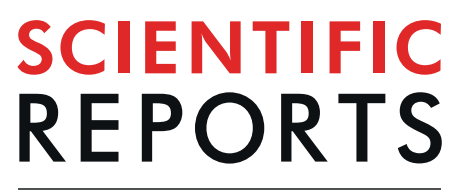

natureresearch

Received: 22 May 2019

Accepted: 21 August 2019

Published online: 20 September 2019

\title{
All-silicon reconfigurable
} metasurfaces for multifunction and tunable performance at optical frequencies based on glide symmetry

\author{
Mohammad Mahdi Shanei $\mathbb{D}^{1,2}$, Davood Fathi $\mathbb{D}^{1,3}$, Fatemeh Ghasemifard $\mathbb{D}^{2}$ \& \\ Oscar Quevedo-Teruel (iD ${ }^{2}$
}

Dielectric metasurfaces have opened promising possibilities to enable a versatile platform in the miniaturization of optical elements at visible and infrared frequencies. Due to high efficiency and compatibility with CMOS fabrication technology, silicon-based metasurfaces have a remarkable potential for a wide variety of optical devices. Adding tunability mechanisms to metasurfaces could be beneficial for their application in areas such as communications, imaging and sensing. In this paper, we propose an all-silicon reconfigurable metasurface based on the concept of glide symmetry. The reconfigurability is achieved by a phase modulation of the transmitted wave activated by a lateral displacement of the layers. The misalignment between the layers creates a new inner periodicity which leads to the formation of a metamolecule with a new sort of near-field interaction. The proposed approach is highly versatile for developing multifunctional and tunable metadevices at optical frequencies. As a proof of concept, in this paper, we design a bifunctional metadevice, as well as a tunable lens and a controllable beam deflector operating at $1.55 \mu \mathrm{m}$.

Metasurfaces are a two-dimensional version of metamaterials composed of metallic and/or dielectric sub-wavelength elements called metaatoms. Metasurfaces are commonly used to manipulate the wavefront by locally controlling of the phase, amplitude and polarization ${ }^{1-3}$. Nowadays, transparent dielectric metasurfaces have been gained remarkable attention due to their low non-radiative Ohmic losses at visible and infrared frequencies. The flat nature of metasurfaces offers new possibilities for integration of optical chips ${ }^{4-6}$.

It has been demonstrated that silicon metaatoms as constitutional elements enhance the efficiency of metasurfaces at infrared wavelengths and can be fabricated in one lithographic step. In addition, they are compatible with complementary metal oxide semiconductor (CMOS) technology ${ }^{7,8}$. The main challenge of designing a high performance metasurface for any application is providing $2 \pi$ phase shifts by means of metaatoms. Silicon resonators with high refractive index supports different orders of the electric and magnetic Mie-type resonances in the optical spectral range. The spectral position of these localized resonances can be tuned by an adjustment of the resonator's geometrical dimensions. More importantly, an overlapping between the first order of the electric and magnetic resonances, which is known as Kerker condition, can be achieved by fine-tuning of the metaatom dimensions. The overlapping of resonance modes produces a $2 \pi$ phase shift together with a near-unit amplitude of the transmitted wave $e^{9-13}$.

Tunable structures have been proposed to make metasurfaces more versatile. For example, tuning mechanisms can enhance the performance of many metadevices such as sensors ${ }^{14-16}$, filters ${ }^{17}$, beam deflectors ${ }^{18}$, absorbers $^{19,20}$, spin-based plasmonic devices ${ }^{21,22}$, optical launchers ${ }^{4,23}$ and lenses ${ }^{24,25}$. Moreover, a dynamic control of the

\footnotetext{
${ }^{1}$ Nanomaterials Group, Department of Materials Engineering, Tarbiat Modares University (TMU), P.O. Box 14115-143, Tehran, Iran. 'Division of Electromagnetic Engineering, KTH Royal Institute of Technology, SE-11428, Stockholm, Sweden. ${ }^{3}$ Department of Electrical and Computer Engineering, Tarbiat Modares University (TMU), P.O. Box 14115-194, Tehran, Iran. Correspondence and requests for materials should be addressed to D.F. (email: d.fathi@modares.ac.ir)
} 
metasurface can be used to switch the function of the device ${ }^{26-28}$. Various approaches have been considered in the literature, such as micro/nanoelectromechanical systems (MEMS/NEMS) ${ }^{24-26,29-32}$, lumped-elements ${ }^{33}$, stretchable substrate ${ }^{34}$, variable carrier densities and effective masses of semiconductors ${ }^{35-38}$, applying a thermo-optics stimulus $^{39,40}$ and phase changes of the materials ${ }^{41,42}$. The latter includes chalcogenide glasses ${ }^{43}$, strongly correlated materials (SCMs) ${ }^{44}$ and liquid crystals (LCs) ${ }^{45,46}$.

To enable a dynamic, fast, continuous and real-time tuning, reconfigurable metasurfaces based on MEMS/ NEMS have been recently studied ${ }^{24,31,32,47}$. The reconfigurability via these technologies is activated by changing the shape of the metaatoms and/or relocating the position of two separated metasurfaces ${ }^{48,49}$. This can be achieved by considering different engineered phase functions for each metasurface. Then, the structures can be reconfigured through a rotation ${ }^{30}$ or a displacement that can be either lateral ${ }^{25}$ or axial $^{24}$. Since the local and selective tuning of the phase modulation by mechanical actuators at nanoscale is challenging, only few works have been carried out in the optical regime, in contrast with the numerous works in $\mathrm{THz}$ and lower frequency ranges ${ }^{32,34,50}$.

In this report, we present a new approach to activate a tuning mechanism in a metasurface composed of two identical layers of silicon blocks (metaatoms) which are separated by a gap. The unit cell of the proposed metasurface creates novel properties that are exquisitely sensitive to displacements between the two layers. Therefore, the tunability is achieved by applying a translation between the two layers. In case the translation is equal to half of the periodicity, the metasurface possesses glide symmetry. This means that the structure is invariant under a translation of half a period and a reflection with respect to the glide plane located in the middle of the gap between the layers. Glide symmetry has been previously proposed to effectively engineer the equivalent refractive index and frequency dispersion behavior of metasurfaces in the microwave regime ${ }^{51,52}$, including studies of the dispersion of surface plasmons ${ }^{53,54}$. However, its effects have never been reported in the optical regime.

Here, glide symmetry is proposed to tailor the spectral position of the resonance frequency and control the phase response of metasurfaces at optical frequencies. We demonstrate that glide symmetry adds an extra structural degree of freedom to the metasurface and results in a construction of a deformable metamolecule which is a combination of several metaatoms within a unit cell. In fact, the displacements between the layers manipulate the near-field interaction between the metaatoms and modifies the spectral position of the excited resonances in the metamolecule. Therefore, this technique is capable of providing a selective sub-wavelength phase modulation of the transmitted wave through the structure. In addition, in the proposed metamolecule, apart from Mie multipoles, another family of resonance, toroidal dipole, has been excited due to the existence of the near-field interaction among metaatoms ${ }^{55}$.

The applied translation vector between the layers imparts an engineered localized phase modulation to metasurfaces which enables a mechanism to tune their operation properties. This selective modulation provides a new degree of freedom that can be employed to design the tunable multifunctional devices. The required translation and dynamic control of the two-layer structure can be achieved by microelectromechanical systems designed for horizontal movements ${ }^{24,56-61}$. For moving parallel plates in the range of micrometers with nanoscale resolution, various actuation mechanisms, such as electrostatical ${ }^{62,63}$, thermal $^{64,65}$ and electrical ${ }^{66}$ have been developed.

The main advantage of the proposed technique is simplicity in design and fabrication that can be controlled with MEMS technology. In the proposed approach, only silicon has been used as the constitutional element of the metasurface, therefore our structure is fully compatible with CMOS technology. Moreover, the structure possesses a high level of tunability associated to the changes of the controllable layers. Therefore, our proposed technique is suitable for ultra-compact devices. As a proof of concept, a tunable metalens and a beam deflector are designed and their performance is presented. In this paper, we study the effects of the gap size inside a metaatom at the frequency of the excited resonances, as well as the effects of adding an extra symmetry to the unit cell. Also, we investigate the resonance modes and transmitted phase responses of the two-layer metasurface with different translation vectors. These translation vectors have been used to design a tunable metalens and a controllable beam deflector. Finally, we employ the reconfigurable metasurface to design a bifunctional metasurface.

\section{Results}

Glide-symmetric unit cell. In this section, we introduce a double-layer unit cell and investigate the effects of different gap sizes between the layers. In addition, the effects of different displacements on the unit cell are studied in the transmittance spectrum.

Figure 1(a) shows the reference unit cell, which is composed of two aligned squared silicon blocks (metaatoms) with length $a$, deposited on $\mathrm{SiO}_{2}$ substrate with permittivity of 2.2 . The gap between the substrates, denoted by $s$, is fixed at $280 \mathrm{~nm}$. However, the gap between the metaatoms, denoted by $g$, is variable. The dielectric function of the silicon metaatom at the working wavelength $\lambda_{0}=1.55 \mu \mathrm{m}$ is obtained from the experimentally measured data in ${ }^{67}$. The periodicity of unit cell is set to $P=1.1 \mu \mathrm{m}$, which is large enough to minimize the near-field interaction among neighboring unit cells. Figure 1(b) represents the transmittance spectrum of the reference unit cell for $t=500 \mathrm{~nm}, a=750 \mathrm{~nm}$ and different gap sizes.

The nature of the Mie resonances is revealed by studying the enhancement of the electric and magnetic field distributions inside the metaatoms. The light-matter interaction for the case of silicon blocks at the wavelength of $1.55 \mu \mathrm{m}$ was previously investigated $\mathrm{in}^{5}$. In Fig. 1(b), as the gap size between the layers increases, a red shift occurs in the resonance wavelength due to the reduction in the effective thickness of the metaatom. The maximum gap that ensures an efficient coupling between the layers is $50 \mathrm{~nm}$. For gap sizes higher than $50 \mathrm{~nm}$, the metaatom of each layer acts as an individual resonator and could not support the lowest order of the electric and magnetic resonances ${ }^{68}$. As shown in Fig. 1(b,c), for the case of $g=80 \mathrm{~nm}$, the resonance dips in the transmittance spectrum and the coupling between the layers are faded. The existence of both electric and magnetic resonances is inevitable for providing the Kerker condition to achieve a $2 \pi$ phase agility. In Fig. 1(c), the normalized intensities of the $x$-component of the electric field distribution for three different gap sizes at their corresponding electric 
a)

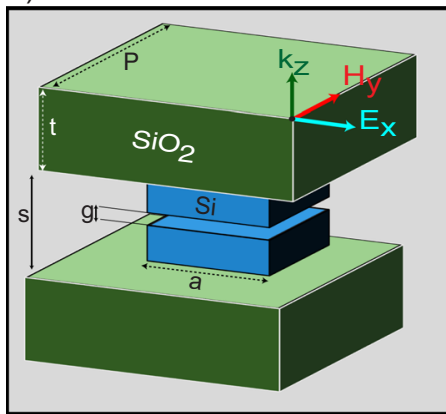

b)

c)

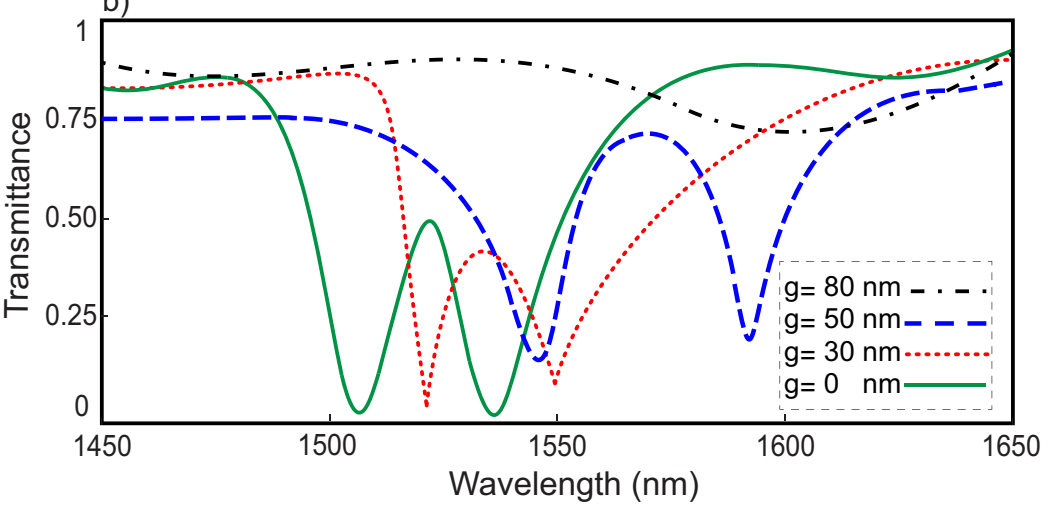

Figure 1. (a) Schematic of the double-layer unit cell (reference unit cell). (b) Transmittance spectrum for different gap sizes. (c) Normalized intensity of the $x$-component of the electric field at the electric resonance frequency for three gap sizes. To obtain $(\mathbf{b}, \mathbf{c}) P=1100 \mathrm{~nm}, t=500 \mathrm{~nm}$ and $a=750 \mathrm{~nm}$ are assumed, while a $x$-polarized plane wave impinges normally on the periodic unit cell.

resonance are shown. For $g=50 \mathrm{~nm}$, in Fig. 1(b), the intensity of the confined resonance decreases due to the reduction of the effective permittivity in the surrounding medium. In addition, the confinement profile of the resonance is more stretched than the case without a gap. For the case with $g=80 \mathrm{~nm}$, since each layer excites its own individual resonance, the confinement profile of the electric field is located inside the lower metaatom.

Figure 2(a) introduces six different translation vectors applied to the reference unit cell (shown in Fig. 1(a)) and illustrates their corresponding unit cells. These unit cells are categorized based on their displacements along the $x$ and $y$-directions. Unit cell type 1 has a zero translation vector. Thus, it is exactly the reference unit cell. Different compositions of a $\frac{P}{4}$ and a $\frac{P}{2}$ shifting in both the $x$ and $y$-directions are applied for other types. Note that the glide symmetry is achieved by alf-period translation. Therefore, unit cell type 3 and type 4 only has the glide symmetry along the $\mathrm{y}$ and $\mathrm{x}$-directions; and unit cell type 6 has glide symmetry in both the $x$ and $y$-directions. For clarity, Fig. 2(b) depicts a 3D view of the glide-symmetric unit cell type 6 and how it is obtained from the reference unit cell. These displacements between the layers construct an effective medium that creates a new inner periodicity in the unit cells. Due to the existence of this inner periodicity and the reduction of lateral distances among blocks, a metamolecule which consists of several metaatoms is formed with a new sort of near-field coupling among metaatoms $\mathrm{s}^{6,70}$. Apart from Mie-types modes, we demonstrate the existence of a toroidal response in the nonaligned unit cell originated from a mutual coupling between adjacent metaatoms in the unit cell. Various configurations of the metamolecules have been investigated so far to excite the toroidal moment ${ }^{71-75}$. Typically for dielectric metasurfaces, the ability of structures in exciting toroidal and Mie-type resonances are studied with the transmittance spectrum and the field distributions at the resonance frequencies. Figure 3(a) illustrates the transmittance spectrum for the unit cells of types $4-6$ with $a=950 \mathrm{~nm}$. The transmittance data of the unit cells type 1-3 are provided in the Supplement S1. For the unit cell type 4, in which $T(x, y)=\frac{P}{2} \hat{x}$, the dips at $1520 \mathrm{~nm}$ and $1580 \mathrm{~nm}$ correspond to the electric and magnetic resonance modes. For the unit cell type $5, T(x, y)=\frac{P}{2} \hat{x}+\frac{P}{4} \hat{y}$, three resonances are excited at $\lambda_{1}, \lambda_{2}$ and $\lambda_{3}$ around our operation wavelength. Finally, for the unit cell type $6, T(x, y)=\frac{P}{2} \hat{x}+\frac{P}{2} \hat{y}$, only the first order of the magnetic resonance is excited in the frequency range of interest. In the unit cell type 5 , a Fano line shape resonance together with two other symmetric resonances are observed in the transmittance spectrum. Figures $3(\mathrm{~b}, \mathrm{c})$ represent the electric field and magnetic field intensity at the magnetic resonance $\lambda_{1}$. The vortex electric field in Fig. 3(b) and the enhanced magnetic field intensity in Fig. 3(c) demonstrate that a magnetic dipole is excited. The electric field distribution pattern and the intensity of the magnetic field, which are shown in Fig. 3(d,e), demonstrate that the second dip is related to an electric quadrupole. At $\lambda_{3}$, the vortex $y$-component of the magnetic field distribution in Fig. 3(f) shows a closed circular pattern penetrating in the upper and lower layers of the metamolecules. As investigated $\mathrm{in}^{70}$, the vortex magnetic field is produced by poloidal current modes. Figure $3(\mathrm{~g})$ shows the $x$-component of the 
a)

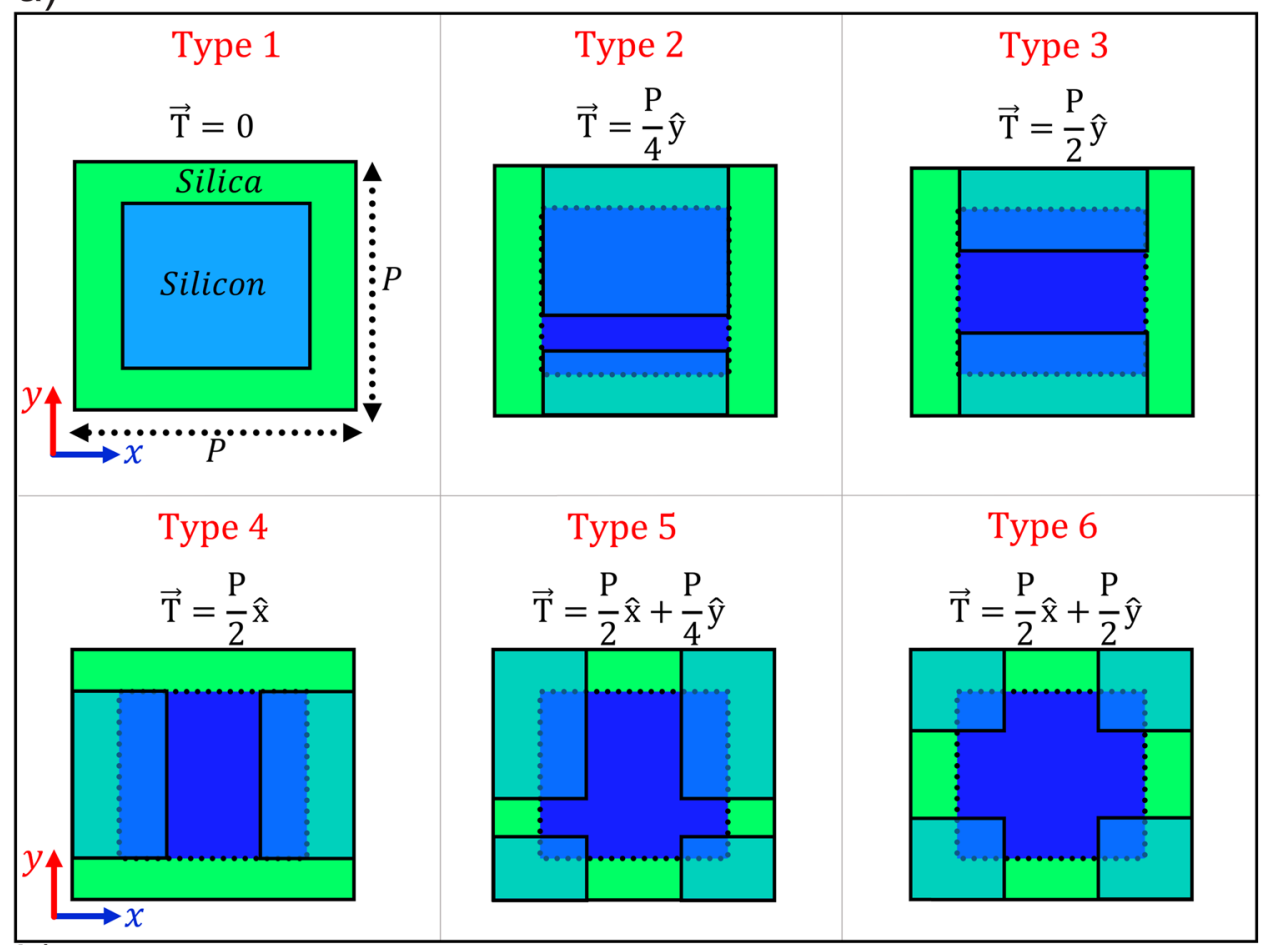

b)

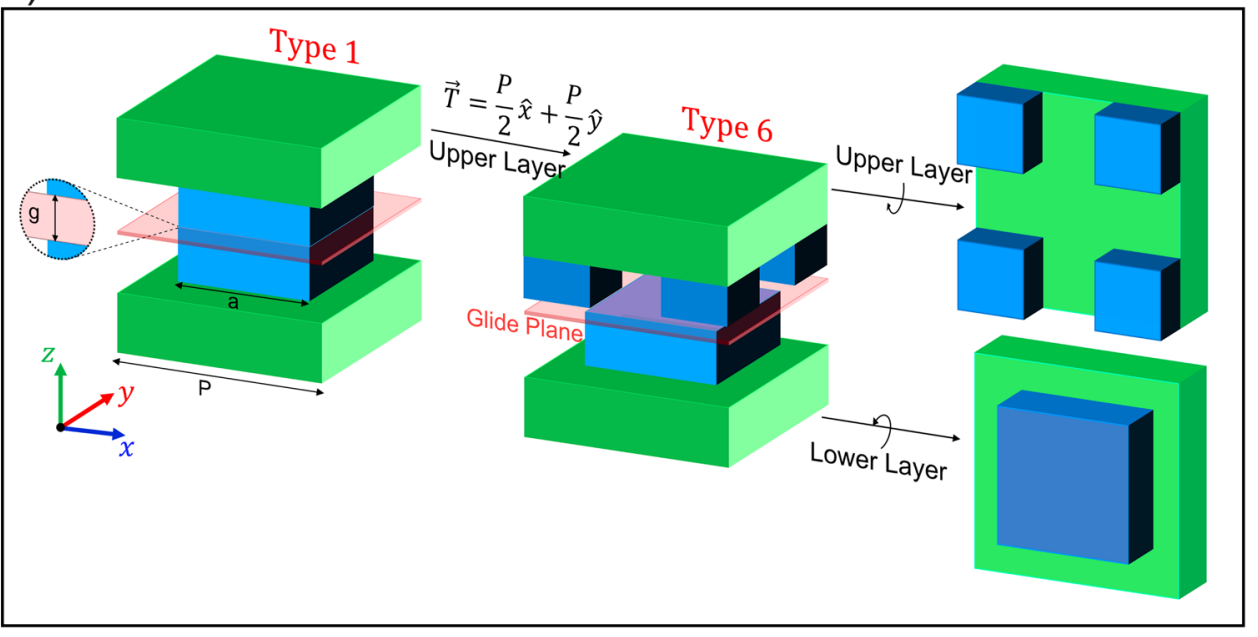

Figure 2. (a) Schematic of the six different types of unit cells achieved by applying the translation vectors to the reference unit cell shown in Fig. 1(a). (b) 3D view of the unit cell type 6 and how it is obtained from the reference unit cell (unit cell type 1).

electric field intensity, which confirms a toroidal electric dipole confined in the gap of the metamolecule. Figure 3(h) schematically depicts the metamolecule supporting a toroidal resonance.

To design a discrete tunable metasurface, one needs to fulfill the corresponding phase profile to control the operation of the device. In our case, we apply different engineered phase modulations by implementing translation vectors. In other words, each performance or functionality is achieved with each of the proposed unit cells in Fig. 2(a). In the designed unit cells, we deliberately consider the existence of different resonances around our operating frequency to produce abrupt changes in the phase response of the transmittance spectrum. In addition, in some cases, the inner periodicity of the unit cells could provide the phase matching conditions for an adequate coupling of the incident wave to guided modes. Therefore, the phase responses of the unit cells are completely decoupled for the different amount of displacement between the layers. Also, each unit cell with the gap size of $50 \mathrm{~nm}$ provides multiple 0 to $2 \pi$ phase coverages as a function of the metaatom size at $1.55 \mu \mathrm{m}$ wavelength. Figure $4(a-f)$ show more details about the $2 \pi$ phase agility of each unit cell.

In the designed unit cells, we deliberately consider the existence of several resonances such as different orders of the electric and magnetic Mie and/or toroidal resonances around the operating frequency to produce the discontinuities in the phase response of the transmittance spectrum. In addition, the inner periodicity of the unit 


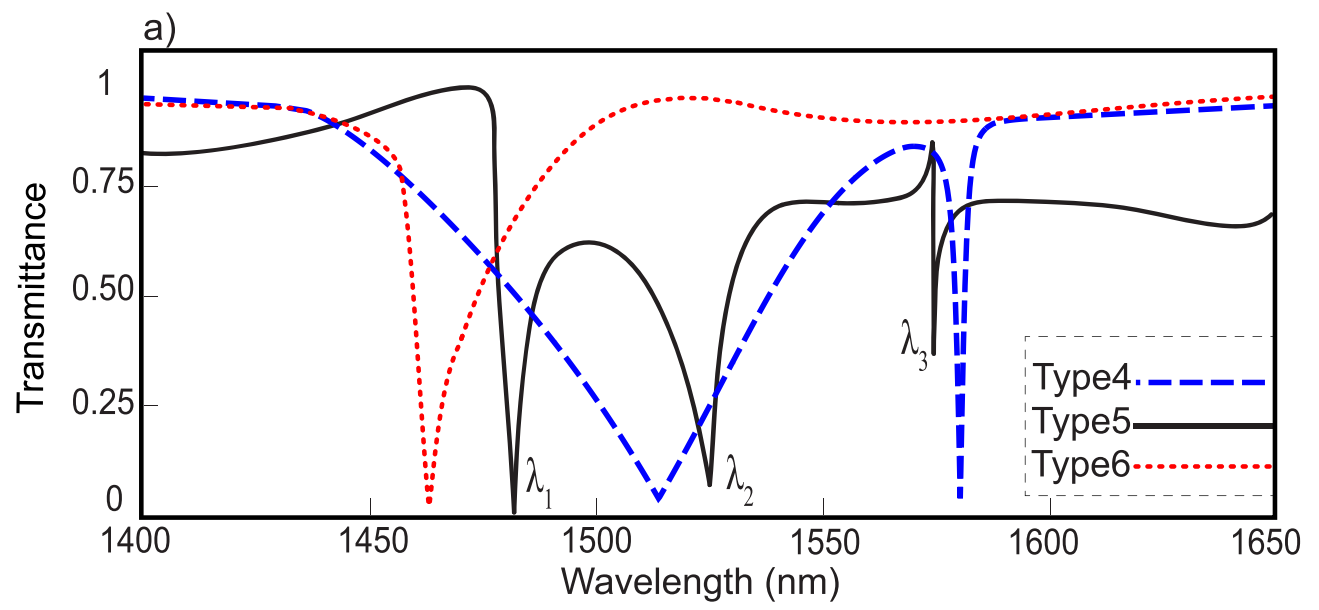

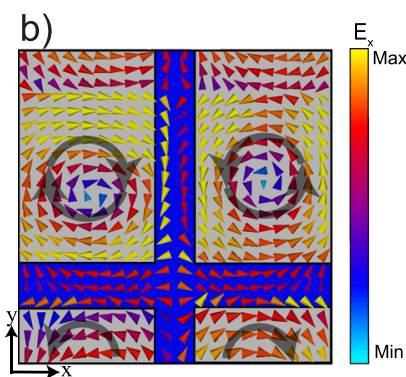

c)

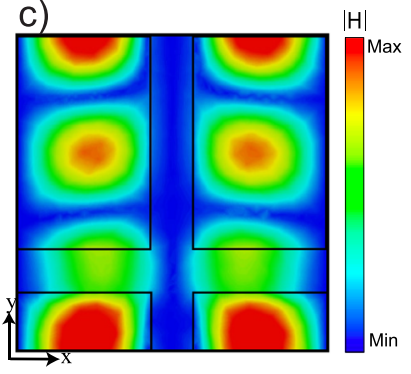

d)

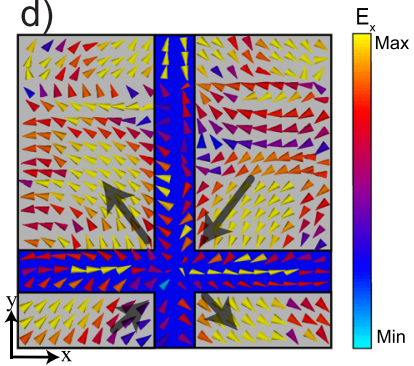

e)

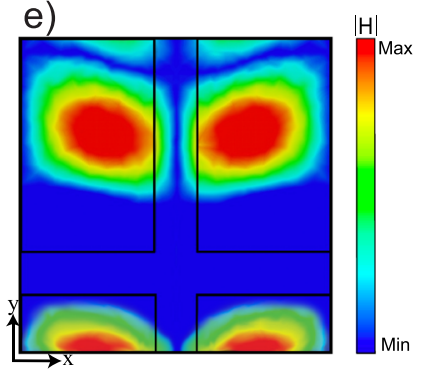

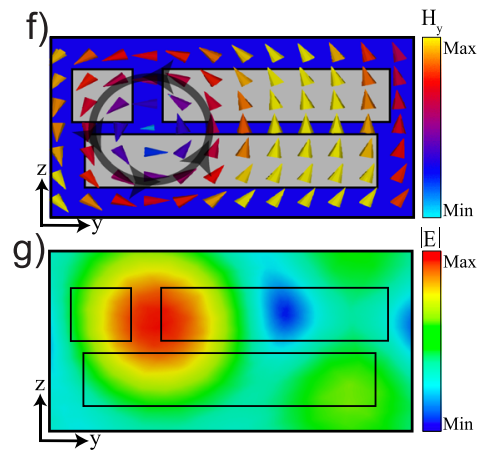

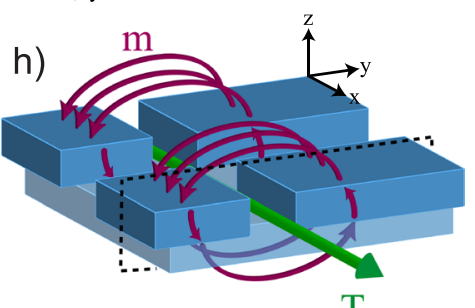

Figure 3. (a) Transmittance spectrum for three types of unit cells with $a=950 \mathrm{~nm}$. $x$-component distribution of the (b) electric field and (c) magnetic field intensity for the unit cell type 5 at $\lambda_{1}=1480 \mathrm{~nm}$. $x$-component distribution of the (d) electric field and (e) magnetic field intensity for the unit cell type 5 at $\lambda_{2}=1530 \mathrm{~nm}$. (f) $y$-component distribution of the magnetic field. (g) Intensity of the electric field at $\lambda_{3}=1573 \mathrm{~nm}$. (h) Schematic of the metamolecule for the unit cell type 5 with the excited electric toroidal resonance.

cells can provide phase matching conditions for an adequate coupling of the incident wave to the guided modes. Therefore, the sharp jumps come from exciting the guided modes in the $x-y$ plane $^{76}$. These resonance modes can effectively introduce multiple abrupt changes in the transmittance phase response.

Lens design. As a first example, we design a tunable line focusing lens with three discrete focal points. Ideally, one would like to have a lens with continuous modification of the focal point. Nevertheless, due to the distinct nature of the decoupled phase responses which is shown in Fig. 4(a-f), we focus here on a discrete modification of the focal point. Since our proposed metalens provides large changes in focal distances with respect to a small variation in the translation vector of $T(x, y)$, this technique could be used to produce bespoke lenses for imaging systems, spectroscopy and integration of optical chips ${ }^{77}$.

The required phase condition for the focal points must be simultaneously fulfilled with a proper arrangement of the metaatoms. The target phase $\phi$ that should be produced by the metaatoms for each position at the metasurface to achieve a constructive interference at a focal point $f$ from the center of the lens is

$$
\phi(x)=\frac{2 \pi}{\lambda_{e f f}}\left(\sqrt{\left(x-x_{f}\right)^{2}+f^{2}}-f\right),
$$

where $\lambda_{\text {eff }}$ is the effective wavelength of the electromagnetic wave, $x$ is the distance from the considered metaatom to the center of the metasurface and $x_{f}$ is the position of the focal point in the transverse direction. 

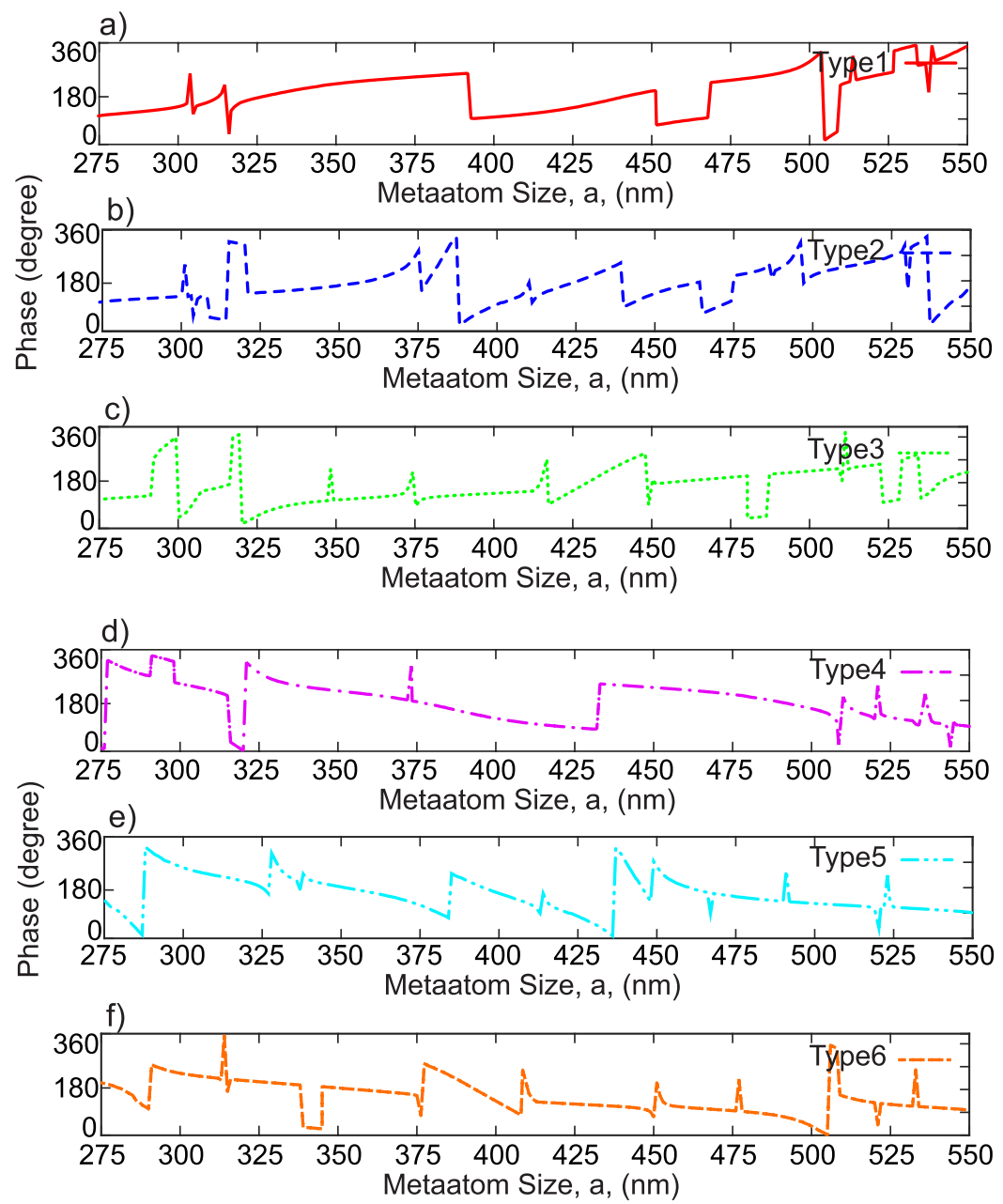

Figure 4. Transmittance phase response of unit cells (a) type 1, (b) type 2, (c) type 3, (d) type 4, (e) type 5 and (f) type 6 as a function of the metaatom size.

There are multiple metaatoms that produce a given phase for each unit cell type. The multiple choices of metaatoms, not only accomplish the phase condition for a lens with high efficiency, but also opens up the possibility of designing tunable metalenses with the ability of changing the distance and direction of the focal points. To design the lenses with the ability of tuning the focal points in axial and diagonal directions, an optimization algorithm has been used. Figure 5(a) represents part of the phase profile needed for three different focal points. The collected data from the transmittance spectrum for unit cells type 1-3 have been plotted based on their phases and amplitudes in Fig. 5(b). In this polar diagram, the rotation shows phases from 0 to $360^{\circ}$ and the radius indicates the transmittance amplitude from 0 to 1 . The phase and amplitude distributions for unit cells type 1, 2 and 3 are shown by triangles, circles and squares, respectively. Also, the colors indicate the metaatom size. In the case of an axial movement of the focal point, we consider unit cell type 1 for the nearest focal point, $f=25 \mu \mathrm{m}$, unit cell type 2 for $f=50 \mu \mathrm{m}$ and unit cell type 3 for $f=75 \mu \mathrm{m}$. The optimization algorithm finds the best metaatom for each position on the surface of the lens to fulfill three phase conditions with three types of unit cells, simultaneously. For instance, in Fig. 5(a), at $x=9 \mu \mathrm{m}$, three target phases should be provided by each metaatom. The algorithm finds the most proper size for the metaatom located at $x=9 \mu \mathrm{m}$. It is worth mentioning that some limitation conditions are applied on the optimization algorithm to pick up the best metaatom with the highest possible transmittance amplitude. The target phases are represented by black stars in Fig. 5(a,b) with black stars. As illustrated in Fig. 5(b), one color, which corresponds to $a=755 \mathrm{~nm}$, has been selected to fulfill the three desired target phases. Therefore, if the displacement between the layers increases from 0 to $\frac{P}{4} \hat{y}$ (unit cell type 2 ) and from $\frac{P}{4} \hat{y}$ to $\frac{P}{2} \hat{y}$ (unit cell type 3 ), the focal point of the lens moves from $25 \mu \mathrm{m}$ to $50 \mu \mathrm{m}$ and from $50 \mu \mathrm{m}$ to $75 \mu \mathrm{m}$. More details about the optimization algorithm and the data preparation for designing the aforementioned metadevice are provided in the Supplement S2.

The required phase conditions for off-axis lenses are calculated by considering three $x_{f}$ distances in Eq. 1. Figure 6(a) depicts the complete phase conditions for the required focal points and the selected phases of the metaatoms. In Fig. $6(\mathrm{~b}-\mathrm{d})$, the field distributions of the lenses are illustrated. Defining the focusing efficiency as the ratio between the optical power focused by the device and the incident power, the focusing efficiencies of the lenses in Fig. 6(b-d) are 
a)

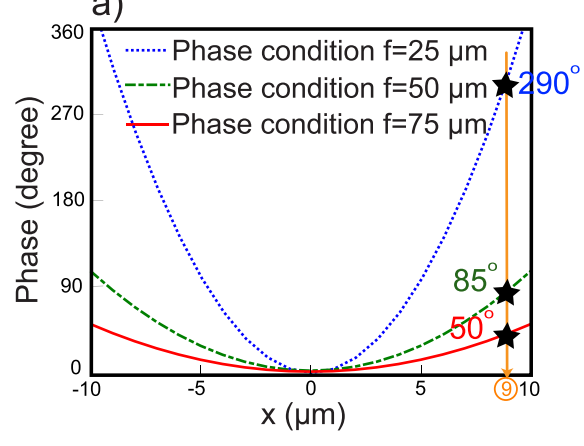

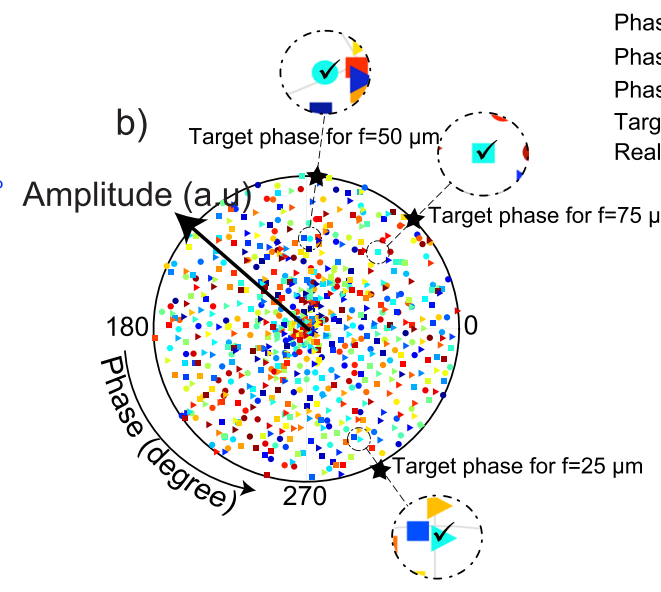

Figure 5. (a) Required phase condition for the three focal points. At $x=9 \mu \mathrm{m}$, black stars show the three required phases. (b) Transmittance phase and amplitude distribution of the realized phase for the three different types of unit cells. The colors illustrate the metaatom sizes, while the geometrical shapes: triangles, circles and squares refer to the data from the unit cell type 1,2 and 3, respectively.
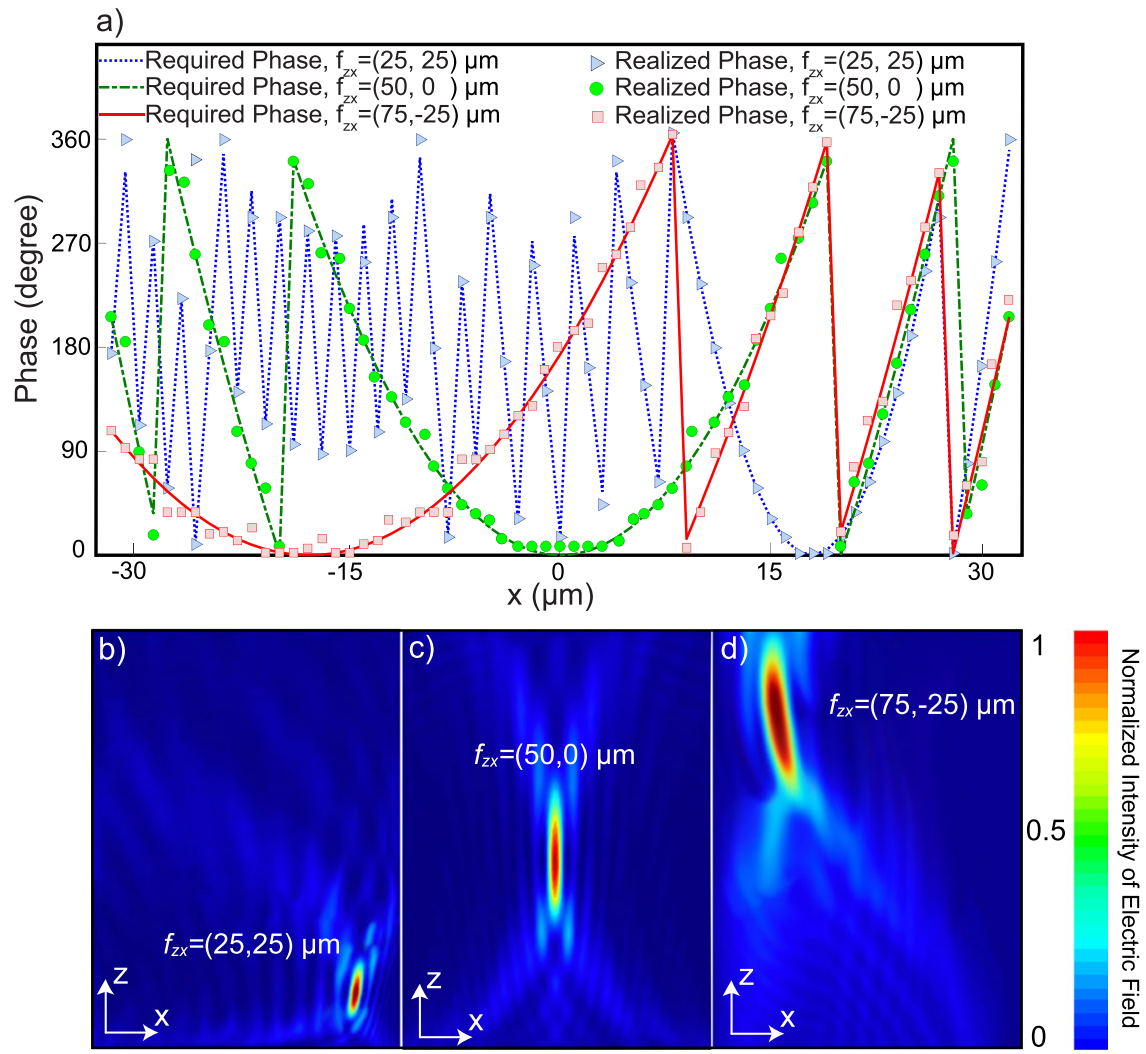

Figure 6. (a) Required phases for the three focal points $f_{z x}=(75,-25) \mu \mathrm{m}, f_{z x}=(50,0) \mu \mathrm{m}$ and $f_{z, x}=(25,25) \mu \mathrm{m}$. Realized phases are shown by triangles, circles and squares for each required phase. The total length of the lens is $71.50 \mu \mathrm{m}$. Normalized intensity of the electric field in the focal plane $x-z$ for $(\mathbf{b})$ $f_{z, x}=(25,25) \mu \mathrm{m}$ achieved by the unit cell type 1 , (c) $f_{z, x}=(50,0) \mu \mathrm{m}$ achieved by the unit cell type 2 and (d) $f_{z, x}=(75,-25) \mu \mathrm{m}$ achieved by the unit cell type 3 . All' intensities are normalized to their maximum value.

$65 \%, 62 \%$ and $56 \%$. The focal points are only switched in the diagonal direction when a shifting in the second layer is produced. In this lens, with a $550 \mathrm{~nm}$ lateral displacement between the layers, the focal point changes to $70 \mu \mathrm{m}$.

Beam deflector design. In order to investigate the capability of double-layer metasurfaces for different applications, in this section, the bilayer metasurface has been employed to steer the impinging electromagnetic waves at three different angles by changing the misalignment between the layers. By considering the Huygens' 


\section{a)}

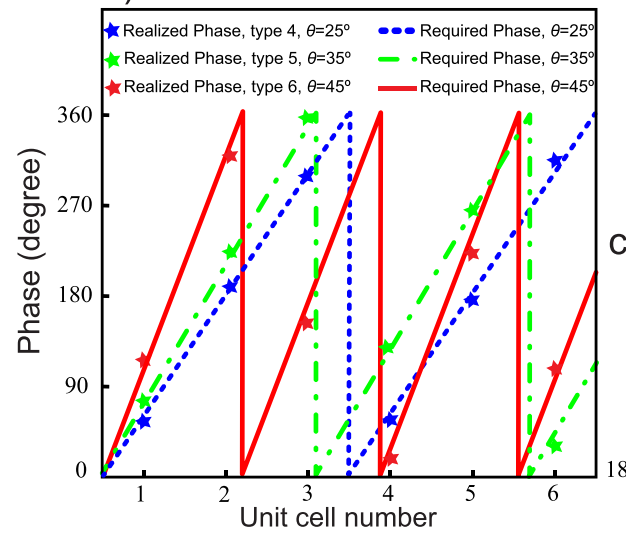

b)

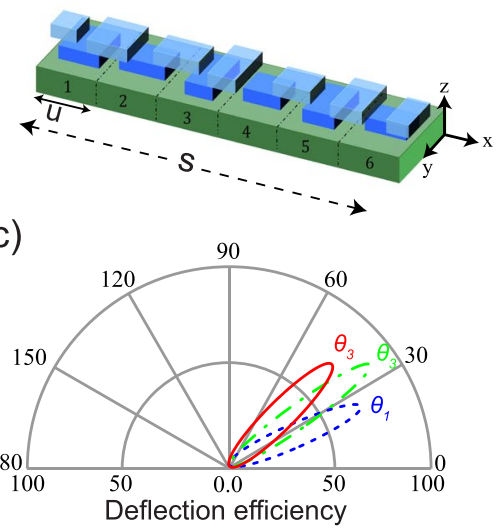

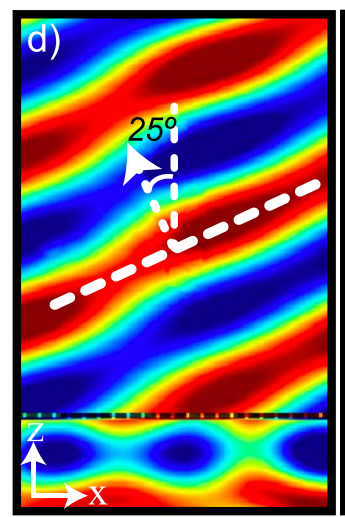
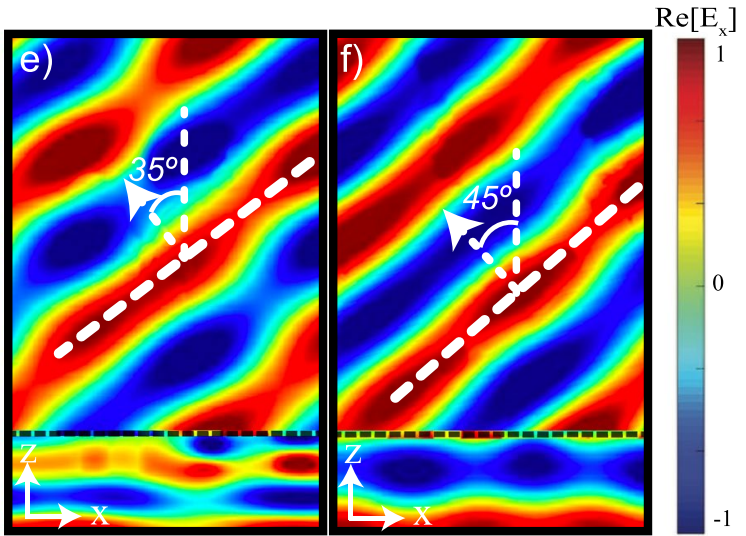

Figure 7. (a) Required phase for deflecting the beam to $\theta_{1}=25^{\circ}, \theta_{2}=35^{\circ}$ and $\theta_{3}=45^{\circ}$. For each one, the realized phases from the unit cells type 4-6 are depicted by stars. (b) Schematic of the super cell with $s=6.6 \mu \mathrm{m}$ and $u=1.1 \mu \mathrm{m}$. (c) Deflection efficiency for $\theta_{1}, \theta_{2}$ and $\theta_{3}$. Distribution of the real part of the $x$-component of the electric field for (d) $25^{\circ}$, (e) $35^{\circ}$ and (f) $45^{\circ}$ in the $x$ - $z$ plane.

principle, an arbitrary steered beam can be constructed by the superposition of spherical wavelets ${ }^{78,79}$. Therefore, based on the generalized Snell's law, a phase gradient can be achieved with a proper arrangement of the metaatoms ${ }^{2}$. The deflection angle for a plane wave can be calculated from,

$$
\sin (\theta)=\frac{\lambda_{0}}{2 \pi} \frac{d \Phi}{\mathrm{d} x}
$$

where $\theta$ denotes the deflection angle of the wave, $\lambda_{0}$ is the incident wavelength, $d \Phi$ is the phase discontinuity between the unit cells and $d x$ is the distance between the center of them.

To design a beam deflector, we consider a super cell configuration that consists of six unit cells. Figure 7(a) shows the required phases to steer the transmitted wave with the deflection angles of $\theta_{1}=25^{\circ}, \theta_{2}=35^{\circ}$ and $\theta_{3}=45^{\circ}$. Again here, an optimization algorithm has been used to find the required size of the metaatom located at the position of $x$ with the ability to provide the required phases with the highest possible transmitted amplitude. The selected metaatom must fulfill the phase condition of Eq. 2 for three desired deflecting angles. In Fig. 7(a), the realized phases from unit cells type 4-6 are shown with stars. The super cell consists of six unit cells of type $4, T(x, y)=\frac{P}{2} \hat{x}$, as depicted in Fig. 7 (b). Figure 7 (c) shows the deflection efficiency defined as the ratio between the deflected power and the total incident power. The deflection efficiency for $25^{\circ}, 35^{\circ}$ and $45^{\circ}$ are calculated as $68 \%, 83 \%$ and $73 \%$ in the $x$ - $z$ plane. The normalized distribution of the real part of the transmitted electric field is shown in Fig. 7(d-f) with the corresponding deflection angle for the unit cells type 4-6. Due to the good matching with the target phases, the deflection angles have a good agreement with the desired deflection calculated with Eq. 2.

Bifunctional metasurface design. Here, using the proposed unit cells, we design a bifunctional metasurface that switches between a lens and a beam deflector. Controlling the functionality is achieved by a $\frac{P}{2}$ translation vector along the $x$-direction (see Fig. 8). When the two-layer structure is aligned along the $x$-direction, a metalens is realized, while $\frac{P}{2}$ misalignment in the $x$-direction results in a deflecting beam with the same structure. The phase functions in Eqs 1 and 2 have been considered in the optimization algorithm to find the best arrangement for minimizing the differences between the required and realized phase for each functionality. Figure 8 shows two performances of a particular arrangement of metaatoms with two different alignments between the layers. The arrangement of the aligned unit cell, type 1 , imposes a hyperbolic phase profile on the metasurface, while a gradient phase shift is introduced across the 


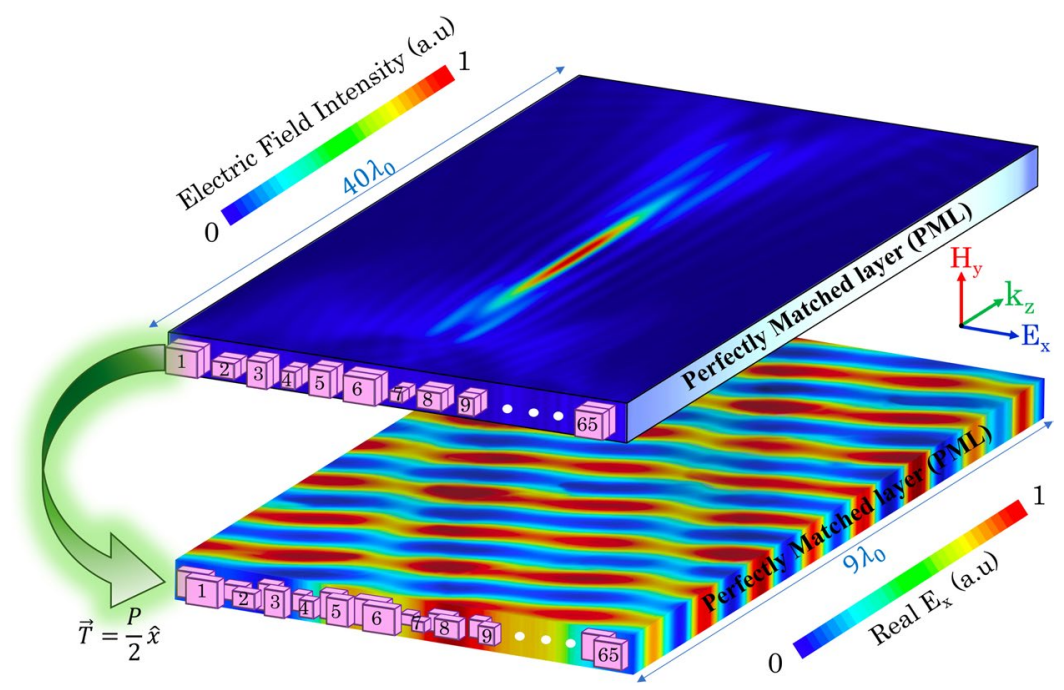

Figure 8. Electric field distribution of a metalens, at the top, with the focal point at $25 \mu \mathrm{m}$ constructed by an aligned two-layer metasurface (unit cell type 1). The distribution of the real part of the $x$-component of the electric field for a beam deflector, at the bottom, with the steering angle of $25^{\circ}$ achieved by applying the translation vector $T(x, y)=\frac{P}{2} \hat{x}$ (unit cell type 4 ) to one layer of the aforementioned metalens. The arrangements of metamolecules have been schematically demonstrated for the metalens with aligned unit cells and for the beam deflector with $T(x, y)=\frac{P}{2} \hat{x}$. To show the performance of two metadevices, the normalized electric field distributions are illustrated in different scales along the $z$-direction.

metasurface by applying the translation vector $T(x, y)=\frac{P}{2} \hat{x}$ between the layers. So, the aforementioned metalens with $f_{z}=25 \mu \mathrm{m}$ switches to a beam deflector with the steering angle of $\theta=25^{\circ}$ as shown in Fig. 8.

\section{Conclusion}

We introduced a reconfigurable metasurface with the selective phase modulation achieved by applying different translation vectors to one layer of the structure. The main purpose of applying translation between the layers, which causes glide symmetry, is to tailor the different orders of excited resonances to enable a local sub-wavelength phase and amplitude modulation.

The misalignments between the layers of a unit cell construct various configurations of metamolecules with a new sort of near-field interaction among the internal metaatoms. These metamolecules can support toroidal resonance rather than the conventional Mie modes. All types of resonances together with the excited guiding modes, provide multiple 0 to $2 \pi$ phase coverage in the transmitted wave as a function of the metaatom size. Indeed, the existence of different modes around our operation wavelength $(1550 \mathrm{~nm})$ builds a phase library for each type of unit cell. These libraries have been utilized to simultaneously fulfill different phase conditions. The proposed technique is general and it can be extended to enhance the functionality and improve the performance of several optical metasurfaces. To demonstrate the capability of the proposed metasurface, we designed a varifocal metalens with three discrete focal points in the diagonal direction and a controllable beam deflector. Also, we re-arranged the metasurface to design a bifunctional metadevice switched via shifting between the layers.

\section{Methods}

In this study, we have performed the full-wave calculations using CST Microwave Studio, which is based on the finite difference time domain (FDTD) method. In all simulations, a hexahedral mesh scheme was used with a minimum mesh size of $15 \mathrm{~nm}$, which is smaller than one-tenth of the effective wavelength. The accuracy level was set at $-70 \mathrm{~dB}$. Simulations assume a linearly $x$-polarized plane wave propagating along the $z$-direction impinging normally on the metasurface. Unit cell boundary conditions were employed for the calculation of the transmittance spectrum. For modeling the line focusing metalens, a periodic boundary condition (PBC) is applied in the $y$-direction and an open boundary condition is set in $x$ - and $z$-directions. To design a tunable beam deflector, a periodic boundary condition is applied in both $x$ - and $y$-directions, while an open boundary condition is adopted in the $z$-direction. Finally, for the simulations of the bifunctional metasurface, a PBC is applied in the $y$-direction and perfectly matched layer (PML) is considered along the $x$-direction.

\section{Data Availability}

The data generated and analysed during the study are available from the authors upon reasonable request.

\section{References}

1. Bao, Y., Jiang, Q., Kang, Y., Zhu, X. \& Fang, Z. Enhanced optical performance of multifocal metalens with conic shapes. Light: Science \& Applications 6, e17071 (2017).

2. Yu, N. et al. Light propagation with phase discontinuities: Generalized laws of reflection and refraction. Science 334, 333-337 (2011). 
3. Arbabi, A., Horie, Y., Bagheri, M. \& Faraon, A. Dielectric metasurfaces for complete control of phase and polarization with subwavelength spatial resolution and high transmission. Nature nanotechnology 10, 937 (2015).

4. Jiang, Q. et al. Spin-controlled integrated near-and far-field optical launcher. Advanced Functional Materials 28, 1705503 (2018).

5. Decker, M. et al. High-efficiency dielectric Huygens' surfaces. Adv. Opt. Mater. 3, 813-820 (2015).

6. Yesilkoy, F. et al. Ultrasensitive hyperspectral imaging and biodetection enabled by dielectric metasurfaces. Nature Photonics 1 (2019).

7. Wu, C. et al. Spectrally selective chiral silicon metasurfaces based on infrared fano resonances. Nat. Commun. 5, 3892 (2014).

8. Shalaev, M. I. et al. High-efficiency all-dielectric metasurfaces for ultracompact beam manipulation in transmission mode. Nano Lett. 15, 6261-6266 (2015).

9. Kerker, M., Wang, D.-S. \& Giles, C. Electromagnetic scattering by magnetic spheres. J. Opt. Soc. Am. A 73, 765-767 (1983).

10. Terekhov, P. D. et al. Enhanced absorption in all-dielectric metasurfaces due to magnetic dipole excitation. Scientific reports 9,3438 (2019).

11. Shanei, M. M., Hashemi, M., Fathi, D. \& Zapata-Rodrguez, C. J. Dielectric metalenses with engineered point spread function. Appl. Opt. 56, 8917-8923 (2017).

12. Wang, S. et al. A broadband achromatic metalens in the visible. Nature nanotechnology 13, 227 (2018).

13. Ou, K. et al. High efficiency focusing vortex generation and detection with polarization-insensitive dielectric metasurfaces. Nanoscale 10, 19154-19161 (2018).

14. Karunasiri, G., Alves, F., Grbovic, D. \& Kearney, B. Identification of nano-scale films for thz sensing. In Micro-and Nanotechnology Sensors, Systems, and Applications IV, vol. 8373, 837326 (International Society for Optics and Photonics, 2012).

15. Tang, W. et al. Dynamic metamaterial based on the graphene split ring high-q fano-resonnator for sensing applications. Nanoscale 8, 15196-15204 (2016).

16. Zhu, Y. et al. Optical conductivity-based ultrasensitive mid-infrared biosensing on a hybrid metasurface. Light: Science \& Applications 7, 67 (2018).

17. Liu, C., Ye, J. \& Zhang, Y. Thermally tunable thz filter made of semiconductors. Optics Communications 283, 865-868 (2010).

18. Komar, A. et al. Dynamic beam switching by liquid crystal tunable dielectric metasurfaces. ACS Photonics 5, 1742-1748 (2018).

19. Andryieuski, A. \& Lavrinenko, A. V. Graphene metamaterials based tunable terahertz absorber: effective surface conductivity approach. Optics Express 21, 9144-9155 (2013).

20. Liu, H., Wang, Z.-H., Li, L., Fan, Y.-X. \& Tao, Z.-Y. Vanadium dioxide-assisted broadband tunable terahertz metamaterial absorber. Scientific reports 9,5751 (2019).

21. Bao, Y. et al. Revealing the spin optics in conic-shaped metasurfaces. Physical Review B 95, 081406 (2017).

22. Ling, X., Zhou, X., Shu, W., Luo, H. \& Wen, S. Realization of tunable photonic spin hall effect by tailoring the pancharatnam-berry phase. Scientific reports 4, 5557 (2014).

23. Yang, H., Cao, G., Ou, K., Li, G. \& Chen, X. Broadband spin-driven anomalous surface plasmon polariton steering via v-shaped aperture metasurfaces. Advanced Theory and Simulations 2, 1800167 (2019).

24. Arbabi, E. et al. MEMS-tunable dielectric metasurface lens. Nat. Commun. 9, 812 (2018).

25. Colburn, S., Zhan, A. \& Majumdar, A. Varifocal zoom imaging with large area focal length adjustable metalenses. Optica 5, 825-831 (2018).

26. Wang, X. et al. Multifunctional microelectro-opto-mechanical platform based on phase-transition materials. Nano letters 18, $1637-1643(2018)$

27. Wen, D., Yue, F., Ardron, M. \& Chen, X. Multifunctional metasurface lens for imaging and fourier transform. Scientific reports 6 , 27628 (2016)

28. Cong, L. et al. Active multifunctional microelectromechanical system metadevices: applications in polarization control, wavefront deflection, and holograms. Advanced Optical Materials 5, 1600716 (2017).

29. Hui, Y., Gomez-Diaz, J. S., Qian, Z., Alù, A. \& Rinaldi, M. Plasmonic piezoelectric nanomechanical resonator for spectrally selective infrared sensing. Nature communications 7, 11249 (2016)

30. Han, J.-H. et al. Rotationally reconfigurable metamaterials based on moire phenomenon. Optics Express 23, 17443-17449 (2015).

31. Zhao, X. et al. Electromechanically tunable metasurface transmission waveplate at terahertz frequencies. Optica 5, 303-310 (2018).

32. Liu, X. \& Padilla, W. J. Dynamic manipulation of infrared radiation with mems metamaterials. Advanced Optical Materials 1, 559-562 (2013)

33. Xu, H.-X., Wang, G.-M., Cai, T., Xiao, J. \& Zhuang, Y.-Q. Tunable pancharatnam-berry metasurface for dynamical and highefficiency anomalous reflection. Optics Express 24, 27836-27848 (2016).

34. Ee, H.-S. \& Agarwal, R. Tunable metasurface and flat optical zoom lens on a stretchable substrate. Nano Lett. 16, 2818-2823 (2016).

35. Gu, J. et al. Active control of electromagnetically induced transparency analogue in terahertz metamaterials. Nature communications 3, 1151 (2012).

36. Li, Z. et al. Active light control of the mos2 monolayer exciton binding energy. ACS nano 9, 10158-10164 (2015).

37. Wang, L. et al. Tailoring active far-infrared resonator with graphene metasurface and its complementary. Plasmonics 12, 353-360 (2017)

38. Kafaie Shirmanesh, G., Sokhoyan, R., Pala, R. A. \& Atwater, H. A. Dual-gated active metasurface at $1550 \mathrm{~nm}$ with wide $\left(>300^{\circ}\right)$ phase tunability. Nano letters 18, 2957-2963 (2018).

39. Lewi, T., Evans, H. A., Butakov, N. A. \& Schuller, J. A. Ultrawide thermo-optic tuning of pbte meta-atoms. Nano letters 17, 3940-3945 (2017).

40. Rahmani, M. et al. Reversible thermal tuning of all-dielectric metasurfaces. Advanced Functional Materials 27, 1700580 (2017).

41. Wang, Q. et al. Optically reconfigurable metasurfaces and photonic devices based on phase change materials. Nature Photonics 10, 60 (2016).

42. Li, X. et al. Switchable multifunctional terahertz metasurfaces employing vanadium dioxide. Scientific reports 9, 5454 (2019).

43. Gholipour, B. et al. Phase-change-driven dielectric-plasmonic transitions in chalcogenide metasurfaces. NPG Asia. Materials 10, 533 (2018)

44. Thompson, Z. J. et al. Terahertz-triggered phase transition and hysteresis narrowing in a nanoantenna patterned vanadium dioxide film. Nano letters 15, 5893-5898 (2015).

45. Atorf, B., Mühlenbernd, H., Muldarisnur, M., Zentgraf, T. \& Kitzerow, H. Electro-optic tuning of split ring resonators embedded in a liquid crystal. Optics letters 39, 1129-1132 (2014)

46. Decker, M. et al. Electro-optical switching by liquid-crystal controlled metasurfaces. Optics Express 21, 8879-8885 (2013).

47. Roy, T. et al. Dynamic metasurface lens based on mems technology. Apl Photonics 3, 021302 (2018).

48. Howes, A., Wang, W., Kravchenko, I. \& Valentine, J. Dynamic transmission control based on all-dielectric huygens metasurfaces. Optica 5, 787-792 (2018).

49. Zheludev, N. I. \& Kivshar, Y. S. From metamaterials to metadevices. Nature materials 11, 917 (2012).

50. Lapine, M. et al. Structural tunability in metamaterials. Applied Physics Letters 95, 084105 (2009).

51. Dahlberg, O., Mitchell-Thomas, R. \& Quevedo-Teruel, O. Reducing the dispersion of periodic structures with twist and polar glide symmetries. Scientific reports 7, 10136 (2017).

52. Ghasemifard, F., Norgren, M. \& Quevedo-Teruel, O. Twist and polar glide symmetries: an additional degree of freedom to control the propagation characteristics of periodic structures. Sci. Rep. 8, 11266 (2018). 
53. Camacho, M., Mitchell-Thomas, R. C., Hibbins, A. P., Sambles, J. R. \& Quevedo-Teruel, O. Designer surface plasmon dispersion on a one-dimensional periodic slot metasurface with glide symmetry. Optics letters 42, 3375-3378 (2017).

54. Quesada, R., Martn-Cano, D., Garca-Vidal, F. \& Bravo-Abad, J. Deep-subwavelength negative-index waveguiding enabled by coupled conformal surface plasmons. Optics letters 39, 2990-2993 (2014).

55. Tasolamprou, A. C., Tsilipakos, O., Kafesaki, M., Soukoulis, C. M. \& Economou, E. N. Toroidal eigenmodes in all-dielectric metamolecules. Phys. Rev. B 94, 205433 (2016).

56. Schaefer, T. M. Lateral motion mems switch. US Patent 6,798,315 (2004).

57. Chen, X., Gao, J. \& Kang, B. Achieving a tunable metasurface based on a structurally reconfigurable array using sma. Opt. Express 26, 4300-4308 (2018).

58. Craighead, H. G. Nanoelectromechanical systems. Science 290, 1532-1535 (2000).

59. Zhang, W.-M., Yan, H., Peng, Z.-K. \& Meng, G. Electrostatic pull-in instability in mems/nems: A review. Sensors and Actuators A: Physical 214, 187-218 (2014).

60. Varona, J., Tecpoyotl-Torres, M. \& Hamoui, A. A. Design of mems vertical-horizontal chevron thermal actuators. Sensors and actuators A: Physical 153, 127-130 (2009).

61. Liu, X., Kim, K. \& Sun, Y. A mems stage for 3-axis nanopositioning. Journal of Micromechanics and Microengineering 17, 1796 (2007).

62. Conrad, H. et al. A small-gap electrostatic micro-actuator for large deflections. Nature communications 6, 10078 (2015).

63. Wang, W. et al. Electrostatic repulsive out-of-plane actuator using conductive substrate. Scientific reports 6, 35118 (2016).

64. Cai, K., Yu, J., Shi, J. \& Qin, Q.-H. Robust rotation of rotor in a thermally driven nanomotor. Scientific reports 7, 46159 (2017).

65. Xu, H. \& Pasini, D. Structurally efficient three-dimensional metamaterials with controllable thermal expansion. Scientific reports 6 , 34924 (2016).

66. Li, X. et al. Nanoporous-gold-based hybrid cantilevered actuator dealloyed and driven by a modified rotary triboelectric nanogenerator. Scientific reports 6, 24092 (2016).

67. Barth, J., Johnson, R., Cardona, M. \& Palik, E. Handbook of optical constants of solids II. (Academic Press, New York, 1991).

68. Liu, X., Fan, K., Shadrivov, I. V. \& Padilla, W. J. Experimental realization of a terahertz all-dielectric metasurface absorber. Opt. Express 25, 191-201 (2017).

69. Marinov, K., Boardman, A., Fedotov, V. \& Zheludev, N. Toroidal metamaterial. New J. Phys. 9, 324 (2007)

70. Tuz, V. R., Khardikov, V. V. \& Kivshar, Y. S. All-dielectric resonant metasurfaces with a strong toroidal response. ACS Photonics $\mathbf{5}$, 1871-1876 (2018).

71. Fang, Z. et al. Removing a wedge from a metallic nanodisk reveals a fano resonance. Nano letters 11, 4475-4479 (2011).

72. Dong, Z.-G., Ni, P., Zhu, J., Yin, X. \& Zhang, X. Toroidal dipole response in a multifold double-ring metamaterial. Opt. Express 20, 13065-13070 (2012).

73. Bao, Y., Hu, Z., Li, Z., Zhu, X. \& Fang, Z. Magnetic plasmonic fano resonance at optical frequency. Small 11, 2177-2181 (2015).

74. Gupta, M. \& Singh, R. Toroidal versus fano resonances in high Q planar THz metamaterials. Adv. Opt. Mater. 4, 2119-2125 (2016).

75. Gupta, M., Srivastava, Y. K., Manjappa, M. \& Singh, R. Sensing with toroidal metamaterial. Appl. Phys. Lett. 110, 121108 (2017)

76. Khorasaninejad, M. et al. Achromatic metalens over $60 \mathrm{~nm}$ bandwidth in the visible and metalens with reverse chromatic dispersion. Nano Lett. 17, 1819-1824 (2017).

77. Ebrahimpouri, M. \& Quevedo-Teruel, O. Bespoke lenses based on quasi-conformal transformation optics technique. IEEE Transactions on Antennas and Propagation 65, 2256-2264 (2017).

78. Baker, B. B. \& Copson, E. T. The mathematical theory of Huygens' principle, vol. 329 (American Mathematical Soc., 2003).

79. Epstein, A. \& Eleftheriades, G. V. Huygens' metasurfaces via the equivalence principle: design and applications. J. Opt. Soc. Am. B 33, A31-A50 (2016).

\section{Author Contributions}

O.Q.T. initiated the idea. O.Q.T., F.G. and M.M.Sh. developed the concept. M.M.Sh. wrote the optimization algorithm and did the calculations and theoretical analysis. O.Q.T. and D.F. supervised the research. All authors discussed the content, analysed the data, reviewed and edited the manuscript.

\section{Additional Information}

Supplementary information accompanies this paper at https://doi.org/10.1038/s41598-019-49395-4.

Competing Interests: The authors declare no competing interests.

Publisher's note Springer Nature remains neutral with regard to jurisdictional claims in published maps and institutional affiliations.

(c) (i) Open Access This article is licensed under a Creative Commons Attribution 4.0 International CC. License, which permits use, sharing, adaptation, distribution and reproduction in any medium or format, as long as you give appropriate credit to the original author(s) and the source, provide a link to the Creative Commons license, and indicate if changes were made. The images or other third party material in this article are included in the article's Creative Commons license, unless indicated otherwise in a credit line to the material. If material is not included in the article's Creative Commons license and your intended use is not permitted by statutory regulation or exceeds the permitted use, you will need to obtain permission directly from the copyright holder. To view a copy of this license, visit http://creativecommons.org/licenses/by/4.0/.

(C) The Author(s) 2019 